\title{
Ferromagnetic transition in diluted magnetic semiconductors
}

\author{
T.Dietl \\ Institute of Physics and College of Science, Polish Academy of Sciences \\ al. Lotników 32/46, PL-02668 Warszawa, Poland
}

Received July 6, 1998

\begin{abstract}
A summary is given of recent theoretical works on effects of the RudermanKittel-Kasuya-Yosida (RKKY) interaction between the localized spins in various dimensionality systems of doped diluted magnetic semiconductors (DMS). Since this interaction is long-range, its influence on the temperature and magnetic field dependencies of magnetization and spin splitting of the bands is evaluated in the mean field approximation, but by taking into consideration disorder-modified carrier-carrier interactions. Theoretical evaluations show that the hole densities, which can presently be achieved, are sufficiently high to drive a paramagneticferromagnetic phase transition in bulk and modulation-doped structures of II-VI DMS. The results of recent magnetooptical studies on MBE-grown samples, containing a single, modulation-doped, $8 \mathrm{~nm}$ quantum well of $\mathrm{Cd}_{1-x} \mathrm{Mn}_{x} \mathrm{Te} / \mathrm{Cd}_{1-y-z} \mathrm{Mg}_{y} \mathrm{Zn}_{z}$ Te: $\mathrm{N}$ are shown to corroborate the theoretical expectations. These studies reveal the presence of a ferromagnetic transition induced by the two-dimensional hole gas. The transition occurs between 1.8 and $2.5 \mathrm{~K}$, depending on the Mn concentration $x$, in agreement with the theoretical model.
\end{abstract}

Key words: semimagnetic semiconductors, RKKY interaction, quantum wells

PACS: $75.50 . R r, 75.30 . H x, 75.50 . D d, 78.55 . E t$

\section{Introduction}

Magnetic properties of diluted magnetic semiconductors (DMS) are known to be dominated by antiferromagnetic superexchange interactions between the localized spins [1]. It has been known for a long time that the compensation of these interactions by a ferromagnetic coupling would result in a dramatic enhancement of the sensitivity of DMS to the temperature and to the magnetic field, particularly in the vicinity of the ferromagnetic phase transition. Search for the ferromagnetic transition has been successful in the case of Mn-based p-type IV-VI [2] and III-V compounds [3], in the latter case a critical temperature as high as $110 \mathrm{~K}$ has recently 
been reported for $\mathrm{Ga}_{0.95} \mathrm{Mn}_{0.05} \mathrm{As}$ [4,5]. In the case of II-VI DMS, a tight binding model [6] suggests that the superexchange in Cr-based DMS might be dominated by a ferromagnetic contribution. Accordingly, an attempt has been undertaken [7] to overcome the well-known small solubility of $\mathrm{Cr}$ in II-VI compounds by means of MBE growth of $\mathrm{Cd}_{1-x} \mathrm{Cr}_{x} \mathrm{Te}$.

A rapid progress in doping of II-VI wide gap semiconductors by substitutional impurities has recently been achieved. For instance, electron and hole concentrations in the excess of $10^{19} \mathrm{~cm}^{-3}$ have been reported for ZnSe:I [8] and ZnTe:N [9]. At the same time, modulation doping of II-VI quantum wells by either electrons [10] or holes [11] as well as patterning of conducting quantum wires [12] has been successfully performed. Motivated by this progress a theoretical analysis of the nature and strength of the carrier-mediated spin-spin interactions in bulk, layered, and nanostructured II-VI compounds has been undertaken by the present authors and co-workers [13]. The results of that work [13] are described here. Two alternative approaches, the RKKY and self-consistent models, are discussed and their equivalence is demonstrated in the mean field approximation (MFA). The role of disorder and carrier-carrier interactions is examined, and shown to be important. Physical arguments supporting the validity of the MFA, even in the reduced dimensionality systems, are also discussed. Quantitative estimations based on the theoretical results are then presented. They indicate that even for the highest available electron density no transition to the ferromagnetic phase is expected above 1 $\mathrm{K}$ in II-VI DMS. By contrast, such a transition is predicted for p-type materials, either in the bulk or modulation-doped form. It has recently been demonstrated $[14,15]$ that indeed the hole liquid in single modulation-doped quantum wells of $\mathrm{Cd}_{1-x} \mathrm{Mn}_{x} \mathrm{Te} / \mathrm{Cd}_{1-y-z} \mathrm{Mg}_{y} \mathrm{Zn}_{z} \mathrm{Te}: \mathrm{N}$ induces the foreseen transition. The corresponding experimental results, together with conclusions and outlook, are presented in the final sections of the present paper. Another review of works devoted to studies of ferromagnetism in II-VI DMS [16] gives a fuller account of the experimental aspects.

The influence of delocalized or weakly localized carriers on the interaction between magnetic ions is of interest here. The dimensionality $d$ of the subsystem of the carriers is determined by the shape of the potential $V(\boldsymbol{\zeta})$ that leads to their confinement. Accordingly, the case $d=1$ or $d=2$ corresponds to a two- or onedimensional potential well, respectively. Because of a short magnetic correlation length [1] the localized magnetic moments $g \mu_{\mathrm{B}} \boldsymbol{S}_{i}$ are assumed, to form a macroscopic 3D system. Thus, according to experimental studies $[1,17]$ their magnetization in the absence of the carriers can be described by a modified Brillouin function, $M(T, H)=g \mu_{\mathrm{B}} \tilde{x} N_{o} S B_{S}\left(T+T_{o}, H\right)$, where effective spin concentration $\tilde{x} N_{o}<x N_{o}$ and temperature $T+T_{o}>T$ account for the influence of antiferromagnetic superexchange interactions $[1,6,17]$.

\section{Ruderman-Kittel-Kasuya-Yosida model}

The carrier-mediated spin-spin coupling is usually described in terms of the Ruderman-Kittel-Kasuya-Yosida (RKKY) model, which provides the energy $J_{i j}$ 
of the exchange coupling, $H_{i j}=-J_{i j} \boldsymbol{S}_{i} \cdot \boldsymbol{S}_{j}$, between two spins located at $\boldsymbol{R}_{i}$ and $\boldsymbol{R}_{j}$ as a function of the density-of-states of the carriers at the Fermi level, $\rho_{d}\left(\varepsilon_{\mathrm{F}}\right)=\pi^{1-d}(2 / \pi)^{(d-2)(d-3) / 2} m^{*} k_{\mathrm{F}}^{d-2} / \hbar^{2}$, and the exchange integral $I$ of their interaction with the spins, $H_{i}=-I \boldsymbol{s} \cdot \boldsymbol{S}_{i} \delta\left(\boldsymbol{R}-\boldsymbol{R}_{i}\right)$. Following the well known procedure [18] and adopting the one-band effective-mass approximation we obtain to the second order in $I$

$$
J_{i j}=\frac{\rho_{d}\left(\varepsilon_{\mathrm{F}}\right) k_{\mathrm{F}}^{d} I^{2}}{2 \pi} \mathcal{F}_{d}\left(2 k_{\mathrm{F}}\left|\mathbf{r}_{i}-\mathbf{r}_{j}\right|\right)\left|\varphi_{o}\left(\boldsymbol{\zeta}_{i}\right)\right|^{2}\left|\varphi_{o}\left(\boldsymbol{\zeta}_{j}\right)\right|^{2} .
$$

Here $\boldsymbol{r}$ is the vector in the $d$ dimensional space, $\varphi_{o}(\boldsymbol{\zeta})$ is the ground-state envelope function of the carriers in the confining potential $V(\boldsymbol{\zeta})$, and

$$
\begin{gathered}
\mathcal{F}_{1}(y)=-\pi \operatorname{si}(y) / 2, \\
\mathcal{F}_{2}(y)=\int_{1}^{\infty} \mathrm{d} t \frac{\mathcal{J}_{1}(y t)}{y t\left(t^{2}-1\right)^{1 / 2}}, \\
\mathcal{F}_{3}(y)=[\sin (y)-y \cos (y)] / y^{4},
\end{gathered}
$$

where $\operatorname{si}(y)$ is the sine-integral and $\mathcal{J}_{n}(y)$ is the Bessel function. The asymptotic behaviour of $\mathcal{F}_{d}(y)$ for large $y$ is $\pi \cos (y) / 2 y, \sin (y) / y^{2}$, and $-\cos (y) / y^{3}$, while for $y \rightarrow 0, \mathcal{F}_{d}$ tends to $\pi^{2} / 4,[1 / 2-\gamma+\ln (4 / y)] / 2$, and $3 / y$ for $d=1,2$, and 3 , respectively, where $\gamma=0.57721 \ldots$ is the Euler constant. For the same sequence of $d$, the first zero of $\mathcal{F}_{d}(y)$ occurs for $y \approx 1.7,3.5$, and 4.5 , respectively. The formula for $d=3$ reproduces the result first obtained by Ruderman and Kittel [19], and for $d=1$ that of Yafet [20], whereas the expression for the case of $d=2$ is equivalent to the formula given recently by Aristov [18]. Knowing the dependence $J_{i j}$ on the distance between the spins $r$ we can calculate the mean-field value of the Curie-Weiss temperature of spins located at $\boldsymbol{\zeta}_{i}, \Theta\left(\boldsymbol{\zeta}_{i}\right)=S(S+1) \sum_{j} J_{i j} / 3 k_{\mathrm{B}}$. Since in semiconductors, unlike in metals, the value of $y$ that corresponds to the distance $r$ between the nearest neighbouring spins is much smaller than the period of the oscillatory functions in equations $2-4, y_{n n}=2 k_{\mathrm{F}} r_{n n} \ll 1$, we replace the summation over the ion positions by an integration extending from $y=0$ to $\infty$. Under the assumption that the distribution of the magnetic ions is random we obtain $\Theta$, averaged over $\boldsymbol{\zeta}$, in the form,

$$
\Theta=S(S+1) N_{o} I^{2} \rho_{d}\left(\varepsilon_{\mathrm{F}}\right) \int \mathrm{d} \boldsymbol{\zeta} \tilde{x}(\boldsymbol{\zeta})\left|\varphi_{o}(\boldsymbol{\zeta})\right|^{4} / 12 k_{\mathrm{B}},
$$

which shows that the net RKKY interaction is ferromagnetic, $\Theta>0$. This is in contrast to the case of metals where a spin glass phase is observed. In semiconductors, however, where the mean distance between magnetic ions $\bar{r}=\left(4 \pi \tilde{x} N_{o} / 3\right)^{-1 / 3}$, is much smaller that the electron wavelength $\lambda_{\mathrm{F}}=2 \pi / k_{\mathrm{F}}$, the spin-spin interaction mediated by the carriers is merely ferromagnetic. Since $\Theta$ is proportional to the effective mass, to the degree of confinement as well as to the square of the exchange integral $I$ we expect much greater magnitudes of $\Theta$ in the presence of the holes than for the electrons in II-VI DMS. 
The above approach neglects intervalley or intersubband virtual transitions. Since those terms in $J_{i j}$, which result from such transitions, contain products of orthogonal envelope functions, $\varphi_{\nu}^{*}\left(\boldsymbol{\zeta}_{j}\right) \varphi_{\nu^{\prime}}\left(\boldsymbol{\zeta}_{j}\right)$, their contribution to $\Theta$ vanishes, provided that the concentration of magnetic ions is uniform. Moreover, in such a case each of the occupied valleys or subbands gives an independent contribution to $\Theta$, described by the relevant $\varphi_{\nu}(\boldsymbol{\zeta})$ and $k_{\mathrm{F}}^{(\nu)}$.

\section{Self-consistent model}

In this section we present results which demonstrate the equivalence - on the level of the MFA and for a random distribution of the magnetic ions - of the RKKY approach and a simple self-consistent Vonsovskii model of the ferromagnetism [22, 23].

We start recalling the molecular-field relation between the magnetization of the localized spins, $M(T, H)$, and the spin-splitting of the relevant band,

$$
\Delta(T, H)=I \int \mathrm{d} \boldsymbol{\zeta} M(T, H, \boldsymbol{\zeta})\left|\varphi_{o}(\boldsymbol{\zeta})\right|^{2} / g \mu_{\mathrm{B}}+g^{*} \mu_{\mathrm{B}} H
$$

where $g$ and $g^{*}$ are the Landé factors of the localized spins and the carrier spins, respectively. In the MFA, $M$ is induced by the external field $H$ and the molecular field produced by the carriers, $H^{*}$ so that $M(T, H)=g \mu_{\mathrm{B}} \tilde{x} N_{o} S B_{S}\left(T+T_{o}, H+H^{*}\right)$, where $H^{*}=I\left(n_{\downarrow}-n_{\uparrow}\right) / 2 g \mu_{\mathrm{B}}$. Here $n_{\downarrow, \uparrow}$ is the density of spin down and spin up carriers, respectively, which, at $\varepsilon_{\mathrm{F}} \gg k_{\mathrm{B}} T$, is given by $n_{\uparrow, \downarrow}=\frac{1}{2}\left|\varphi_{o}(\boldsymbol{\zeta})\right|^{2} \int_{ \pm \Delta / 2}^{\varepsilon_{\mathrm{F}}} \mathrm{d} \varepsilon \rho_{d}(\varepsilon)$, where the dependence of $\varepsilon_{\mathrm{F}}$ on $\Delta$ is to be determined from the condition $n_{\uparrow}+n_{\downarrow}=n$, with $n$ being the total carrier density. A similar model of ferromagnetism, developed for the case of a nondegenerate gas of carriers thermally activated from the impurity levels, was put forward by Pashitskii and Ryabchenko [23].

The above set of coupled equations makes it possible to determine in a selfconsistent way the mean-field values of $\Delta$ as functions of temperature and magnetic field. In particular, in order to evaluate $\Delta(T, H)$ above the phase transition we take into account the terms which are linear in $H+H^{*}$. Such a procedure leads to,

$$
\Delta(T, H)=\frac{\Delta_{o}(T, H)}{\left[1-\frac{I^{2} \rho_{d}\left(\varepsilon_{\mathrm{F}}\right)}{4 g^{2} \mu_{\mathrm{B}}^{2}} \int \mathrm{d} \boldsymbol{\zeta} \chi_{o}(T, \boldsymbol{\zeta})\left|\varphi_{o}(\boldsymbol{\zeta})\right|^{4}\right]},
$$

where $\Delta_{o}(T, H)$ and $\chi_{o}(T)$ are the spin-splitting and the magnetic susceptibility in the absence of the free carriers.

We see that for the magnetic susceptibility of the form $\chi_{o}(T)=C /\left(T+T_{o}\right)$, $\Delta(T)$ diverges at $T_{\mathrm{c}}=\Theta-T_{o}$, where $\Theta$ turns out to coincide with the Curie-Weiss temperature determined by the RKKY interactions, displayed in equation 5 .

\section{Applicability of the mean-field approximation}

The long range character of the ferromagnetic interaction, $\lambda_{\mathrm{F}} \gg \bar{r}$, has also important consequences for the nature of the phase transition. It has been shown 
[24] that as long as $\sigma<d / 2$ in the dependence $J(r) \sim 1 / r^{d+\sigma}$, the mean-field approach to the long wavelength susceptibility $\chi(T)$ is valid, a conclusion non-affected presumably by disorder in the spin distribution. By contrast, the critical exponents [24] $\eta=2-\sigma$ and $\nu=1 / \sigma$ point to much faster decay of $\chi(q)$ with $q$ than that expected from the classical Ornstein-Zernike theory [25]. This means that our meanfield model of the ferromagnetic transition driven by the RKKY interactions should remain valid down to at least $\left|T-T_{\mathrm{c}}\right| / T_{\mathrm{c}} \approx\left(\bar{r} k_{\mathrm{F}}\right)^{2}$. At the same time, unlike the case of short range interactions, the length scale of magnetic correlation is set by $\lambda_{\mathrm{F}}$, not by $\bar{r}$. This means that for $\lambda_{\mathrm{F}} \gg \bar{r}$ the critical fluctuations of magnetization will be strongly suppressed, an expectation corroborated by the virtual absence of critical scattering of the carriers by the Mn spins in bulk $\mathrm{Pb}_{1-x-y} \mathrm{Mn}_{x} \mathrm{Sn}_{y} \mathrm{Te}$ [26], where the free holes drive a ferromagnetic transition [2], and its presence in, e.g., EuS: Gd and EuO:Gd [27], in which a short range ferromagnetic interaction between the Eu spins dominates [28]. At the same time, it is expected that the suppression of the critical fluctuations will make it possible to probe directly the magnetic long range order by means of inter- or intraband magnetospectroscopy.

\section{Effects of disorder, carrier-carrier interactions, and magnet- ic polarons}

So far we have disregarded the influence of the potential scattering and the Coulomb interactions among the carriers upon the magnitude of $\Theta$. The former is known [29] to introduce a random phase shift in the oscillatory functions of equation 1. This random phase shift has no effect on the magnitude of the second moment of the distribution of $J_{i j}$ [30] but, after averaging over the disorder, leads to the dumping $\exp (-r / \ell)$ of the first moment $[29,30]$, where $\ell$ is the mean free path for elastic collisions. The corresponding reduction factor of $\Theta$ to the lowest order in $1 / k_{\mathrm{F}} \ell$ is given by $1-1 / 12\left(k_{\mathrm{F}} \ell\right)^{2}, 1-\pi / 8 k_{\mathrm{F}} \ell$, and $1-\pi / 4 k_{\mathrm{F}} \ell$ for $d=1,2$, and 3 , respectively. Hence the effect of disorder becomes important on approaching the strongly localized regime, $k_{\mathrm{F}} \ell \rightarrow 1$. In this range, however, the magnetic susceptibility of the carriers becomes substantially enlarged by the disorder-modified electronelectron interactions [31]. The resulting enhancement factor of $\Theta$, originating from the RKKY interactions between the spins located at $r>\ell$ [32], can be written in the form: $A_{\mathrm{F}}=1+F L_{\mathrm{s}} / \ell, 1+2 F \ln \left(L_{\mathrm{s}} / \ell\right) / \pi k_{\mathrm{F}} \ell$, and $1+3 F\left(1-\ell / L_{\mathrm{s}}\right) /\left(2 k_{\mathrm{F}} \ell\right)^{2}$, respectively. Here $F$ is the effective Coulomb amplitude, which becomes greater than 1 for $k_{\mathrm{F}} \ell \rightarrow 1 ; L_{\mathrm{s}}=\sqrt{\left(D t_{\mathrm{s}}\right)} \gg \ell$ is the spin diffusion length, where $D=k_{\mathrm{F}} \ell / d m^{*}$ and $t_{\mathrm{s}}$ is the spin-disorder scattering time [31,33]. The effects of disorder and electronelectron interactions can also be incorporated into the self-consistent formalism by taking into account collision broadening of the density of states as well as by determining $H^{*}$ from $g \mu_{\mathrm{B}} H^{*}=I \partial \Omega / \partial \Delta$ with the Gibbs free energy of the carriers, $\Omega$ containing effects of the disorder-modified electron-electron interactions [31].

A crucial question which arises at this point concerns the actual magnitude of the enhancement factor $A_{\mathrm{F}}$ in the real materials. It turns out that $A_{\mathrm{F}}$ is approximately equal to $1+g_{3}$, where $g_{3}$ is a parameter that controls the spin-splitting-induced 
positive magnetoresistance in disordered systems [31]. For example, by taking $g_{3}$ determined for bulk n- $\mathrm{Cd}_{0.95} \mathrm{Mn}_{0.05} \mathrm{Se}$ [34] and superlattices of $\mathrm{n}-\mathrm{Si} / \mathrm{Si}_{0.5} \mathrm{Ge}_{0.5}$ [35] we obtain $A_{\mathrm{F}} \approx 2.3$ and 2.5 , respectively. Such a mechanism leads also to an enhancement of the spin-disorder scattering rate, as demonstrated by an analysis of the linewidth of spin-flip Raman scattering (SRRS) in $\mathrm{n}-\mathrm{Cd}_{0.95} \mathrm{Mn}_{0.05} \mathrm{Se}$ [36]. By contrast, the interactions within the Fermi liquid do not renormalize the carrier spin-splitting $\Delta(T, H)$, as given by the Stokes shift of the SFRS line [36]. The lack of contribution to $\Delta(T, H)$ from the exchange interactions among the carriers stems from the fact that the projection $S_{z}$ of the total spin $S$ of the carriers undergoes a change in the process of SFRS, while $S$, and thus the interaction energy, remain conserved.

A particularly strong can be a combined effect of disorder and on-site Hubbard repulsion in the vicinity of the metal-to-insulator transition (MIT) in doped semiconductors. Here, a growing amount of evidences has been collected in favour of a phenomenological two-fluid model of electronic states [37]. According to that model the conversion of itinerant electrons into local moments occurs gradually, and begins already on the metal side of the MIT, leading to the coexistence of the extended and strongly localized states. In magnetic materials, the local moments can be polarized, via the $s$ - $d$ interaction, the neighbouring Mn spins (there are about $200 \mathrm{Mn}$ ions within the Bohr orbit in $\mathrm{n}-\mathrm{Cd}_{0.95} \mathrm{Mn}_{0.05} \mathrm{Se}$ ). Bound magnetic polarons formed in this way not only impose a local ferromagnetic order above $T_{\mathrm{c}}$, but also constitute the centers of efficient spin-dependent scattering for itinerant electrons [34,36,38]. The corresponding scattering rate is proportional to the degree of polaron polarization. The latter is proportional to the magnetic susceptibility of the Mn spins, $\chi(T)$, and therefore increases steeply on approaching the ferromagnetic phase transition. This mechanism accounts presumably for critical scattering of holes and negative magnetoresistance detected recently in $\mathrm{p}-\mathrm{Ga}_{1-x} \mathrm{Mn}_{x}$ As near $T_{\mathrm{c}}$ [5]. By contrast, no such effects are seen in p- $\mathrm{Pb}_{1-x-y} \mathrm{Mn}_{x} \mathrm{Sn}_{y} \mathrm{Te}$ [26], where a large dielectric constant reduces the Hubbard repulsion, and thus precludes the formation of bound magnetic polarons.

We conclude that, in fact, the disorder is expected to enlarge a characteristic temperature of the ferromagnetic interactions in semiconductor structures. This enhancement may also be important in pure 1D systems, where the interaction-driven separation of the charge and spin degrees of freedom modifies $J(r)$ [39]. In the zerodimensional case such as quantum dots both correlation effects and the fluctuations of magnetization associated with the finite volume visited by the carriers are of the paramount importance. A variant of the theory developed for bound magnetic polarons [40] should be applied for those systems.

\section{Quantitative estimations}

Figure 1 shows the magnitude of $\Theta(x)$ for p-type and n-type bulk $\mathrm{Cd}_{1-x} \mathrm{Mn}_{x} \mathrm{Te}$, calculated by equation 5 , with the values of $\tilde{x}(x)$ and $T_{o}(x)$, as determined by Gaj et al. [17] at $T=1.7 \mathrm{~K}$ as well as by taking $m^{*}=0.8$ and $0.1 m_{o}$ as well as 


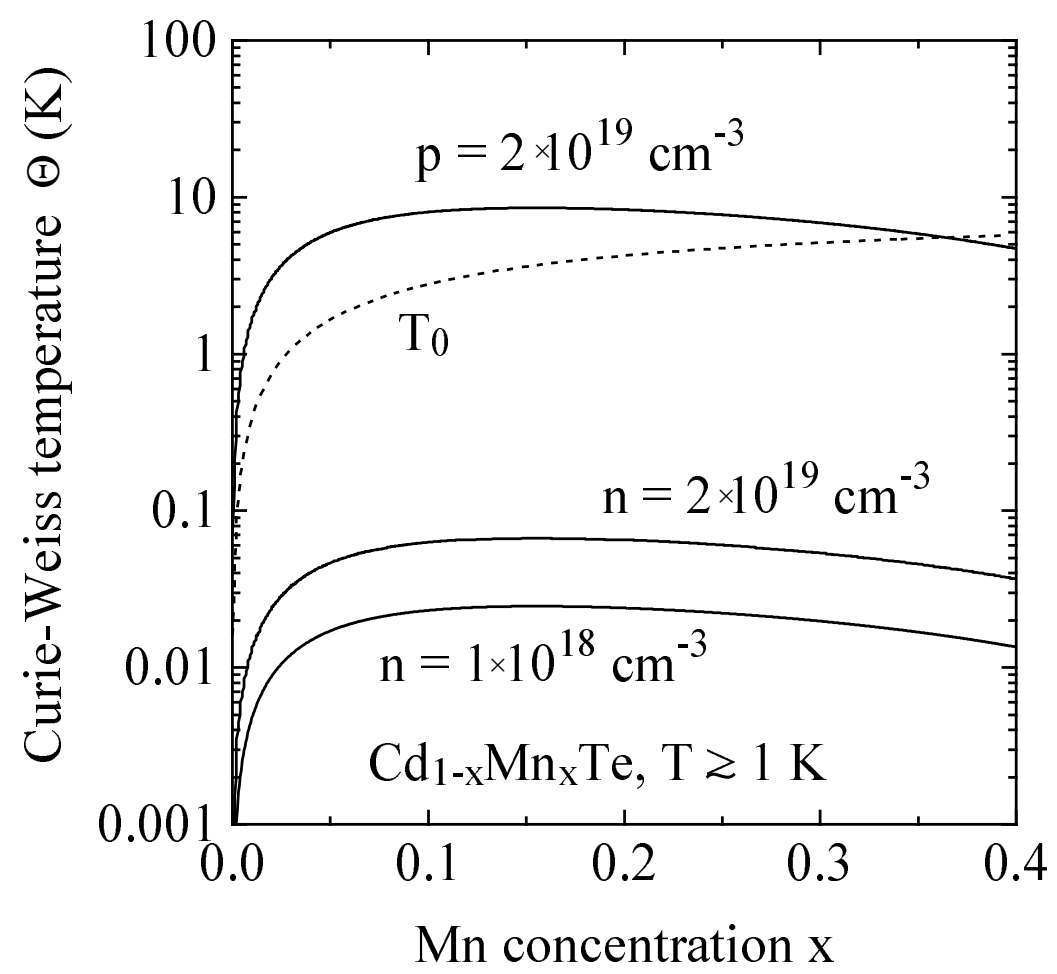

Figure 1. Mean-field value of the Curie-Weiss temperature $\Theta$ calculated from equation 5 for $\mathrm{p}$ - and n-type $\mathrm{Cd}_{1-x} \mathrm{Mn}_{x} \mathrm{Te}$, compared to antiferromagnetic temperature $T_{o}(x)$. Ferromagnetic phase transition occurs at $T_{\mathrm{c}}=\Theta-T_{o}$. Material parameters as determined at $1.7 \mathrm{~K}$ together with the enhancement factor $A_{\mathrm{F}}=1$ were adopted for the calculation (after Dietl et al. [13]).

$I N_{o} \equiv \beta N_{o}=-0.88$ and $I N_{o} \equiv \alpha N_{o}=0.22 \mathrm{eV}$ for the holes and the electrons, respectively $[17,41]$. It has been noted that the spin-orbit interaction reduces the spin-splitting of the $\Gamma_{8}$ heavy holes at the Fermi level according to [42] $\Delta(\boldsymbol{k})=I|\boldsymbol{M} \cdot \boldsymbol{k}| / g \mu_{\mathrm{B}} k$, which after angular averaging results in an effective spin density of states $\tilde{\rho_{\mathrm{F}}}=\frac{1}{2} \rho_{\mathrm{F}}$. We see in figure 1 that for sufficiently high hole concentrations so that the holes remain delocalized [43], $\Theta>T_{o}$ in a wide range of $\mathrm{Mn}$ concentrations. Furthermore, since for the above parameters the Kondo temperature $T_{K} \approx \varepsilon_{\mathrm{F}} \exp \left[-1 /\left(3|I| \tilde{\rho_{\mathrm{F}}}\right)\right]=1.1 \mathrm{~K}$, a crossover to the Kondo regime, $T_{K}>\Theta, T_{o}$ will take place at relatively low Mn concentrations, $x<1 \%$. These considerations suggest, therefore, that a ferromagnetic phase transition can occur above $1 \mathrm{~K}$ in $\mathrm{p}^{+}$ II-VI compounds. This is unlike the case of n-type doping, for which no ferromagnetic phase transition is expected above $1 \mathrm{~K}$, as shown by the two bottom curves in figure 1. It is worth noting, however, that on lowering temperature $T_{o}$ decreases, especially for low Mn concentrations [44]. Indeed, for the highest value of $\chi_{o}$ ever reported for any DMS [44], that is $\chi_{o}=4.8 \times 10^{-3} \mathrm{emu} / \mathrm{g}$ for $\mathrm{Cd}_{0.99} \mathrm{Mn}_{0.01}$ Se at $15 \mathrm{mK}$, a ferromagnetic phase transition is predicted from equation 5 for material parameters of $\mathrm{n}_{-} \mathrm{Cd}_{1-x} \mathrm{Mn}_{x}$ Se [40] provided that $n \geqslant 1.5 \times 10^{19} \mathrm{~cm}^{-3}$. Since $I>0$, there is no Kondo effect for the electrons in DMS.

Turning to the case of holes in DMS quantum wells we note that the confinement 
and possibly the biaxial strain lead to a splitting of the heavy and light hole bands [45] as well as to a strong anisotropy of the spin-splitting [46]. Actually, the coupled system of the 2D holes and the Mn spins is Ising-like as the spin-splitting of the ground-state subband undergoes a maximum for the magnetization parallel to the growth axis. By taking parameters suitable for the uppermost heavy-hole subband in a quantum well of $\mathrm{Cd}_{0.9} \mathrm{Mn}_{0.1} \mathrm{Te}, m_{\mathrm{h}}^{*}=0.25 m_{o}$ and $\left|\varphi_{o}(z)\right|^{2}=1 / L_{\mathrm{W}}$, where $L_{\mathrm{W}}=50 \AA$, we predict the ferromagnetic transition to occur at about $2 \mathrm{~K}$ for $A_{\mathrm{F}}=1$, independently of the hole area concentration $p$, since in our model the 2D density of states does not vary with $\varepsilon_{\mathrm{F}}$. At the same time, the saturation values of $\Delta$ and $M$ do depend directly on $p$ according to $\Delta_{\mathrm{s}}(T)=I M\left(T, H_{\mathrm{s}}^{*}\right) / g \mu_{\mathrm{B}}$, where $H_{\mathrm{s}}^{*}=I p / 2 g \mu_{\mathrm{B}} L_{\mathrm{W}}$, a value of the order of $1 \mathrm{kOe}$ for $p=2 \times 10^{11} \mathrm{~cm}^{-2}$.

Finally, we note that in the case of 1D structures $\Theta$ increases with decreasing the carrier concentration. This, together with the correlation effects discussed above demonstrate the outstanding properties of such systems.

\section{Comparison with experimental results}

The relevant experimental studies [14,15] have been carried out on 2D structures grown by molecular beam epitaxy (MBE) [11]. The nitrogen modulation-doped structures consist of a single $8 \mathrm{~nm}$ quantum well (QW) of $\mathrm{Cd}_{1-x} \mathrm{Mn}_{x}$ Te embedded in $\mathrm{Cd}_{0.66} \mathrm{Mg}_{0.27} \mathrm{Zn}_{0.07} \mathrm{Te}$ barriers grown coherently onto a (001) $\mathrm{Cd}_{0.88} \mathrm{Zn}_{0.12} \mathrm{Te}$ substrate. Such a layout insures large confinement energies for the holes in the QW, minimizing at the same time the effects of lattice mismatch. Nitrogen-doped region in the front barrier is at the distance of $20 \mathrm{~nm}$ or $10 \mathrm{~nm}$ to the QW. Furthermore, in order to reduce depleting effects, two additional nitrogen doped layers reside at the distance of $100 \mathrm{~nm}$ from the QW on both sides. The nominal hole concentrations in the doped structures, evaluated from a self consistent solution of the Poisson and Schrödinger equations, are $2 \times 10^{11} \mathrm{~cm}^{-2}$ and $3 \times 10^{11} \mathrm{~cm}^{-2}$ for the two employed values of the spacer width. In the same way we obtain $\tilde{L}_{\mathrm{W}}=6.3 \mathrm{~nm}$ as an effective width of the hole layer, which is related to the hole ground state envelope function $\varphi_{o}(z)$ by $\tilde{L}_{\mathrm{W}}=1 / \int \mathrm{d} z\left|\varphi_{o}(z)\right|^{4}$. For a control purpose, an undoped structure was also grown and examined.

An examination of the photoluminescence (PL) and its excitation spectra (PLE) was carried out as a function of temperature and the magnetic field $[14,15]$. The magnetic field was parallel to the growth direction and the two circular polarizations in the Farady geometry were employed. As shown in figure 2, the PL splitting $\Delta=\left|E_{-}-E_{+}\right|$under such conditions is not only exceptionally large, but increases in a dramatic way on lowering temperature. Actually, in all doped samples, a colossal value of $\partial \Delta / \partial H$ was observed above a characteristic temperature $T_{\mathrm{c}}$, and a zero field splitting below $T_{\mathrm{c}}$, where $T_{\mathrm{c}}=1.8 \mathrm{~K}$ for $x=0.024$ [14] and $2.5 \mathrm{~K}$ for $x=0.037$ [15]. The corresponding experimental data are summarized in figure 2. No such effects were visible either in the undoped structure or in the presence of illumination by white light that depletes the QW from the carriers. The appearance of zerofield splitting was preceded by a critical increase of $\chi$, as shown in figure $2 \mathrm{~b}$. The 


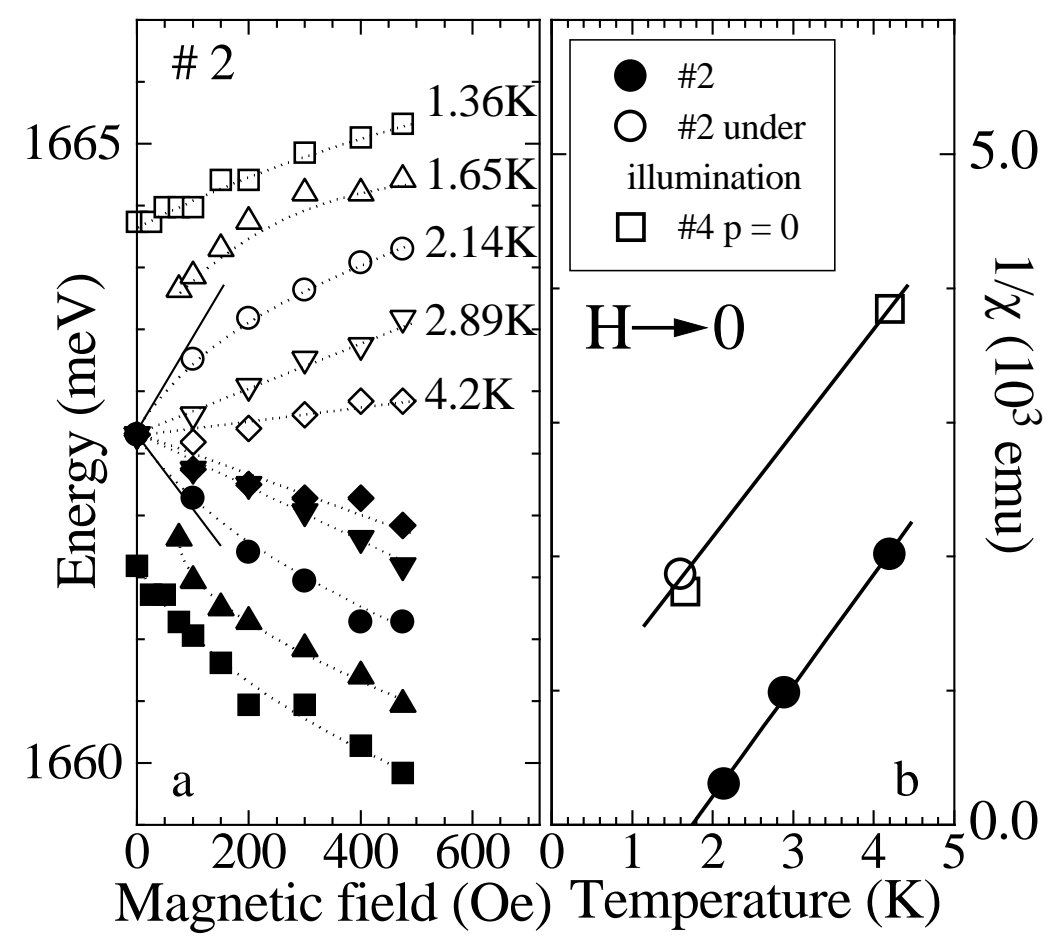

Figure 2. (a) Energy $E_{ \pm}$of photoluminescence maxima at $\sigma^{+}$and $\sigma^{-}$circular polarizations (full and empty symbols, respectively) as a function of the magnetic field $H$ for selected temperatures in a modulation-doped p-type QW of $\mathrm{Cd}_{0.976} \mathrm{Mn}_{0.024} \mathrm{Te}$. Dotted lines are guides for the eye; solid lines denote the assumed initial slope of $E_{ \pm}$at $2.14 \mathrm{~K}$. (b) Inverse magnetic susceptibility calculated from $\mathrm{d}\left(E_{-}-E_{+}\right) / \mathrm{d} H$ at $H \rightarrow 0$, as given by data in (a) for p-type QW of $\mathrm{Cd}_{0.976} \mathrm{Mn}_{0.024} \mathrm{Te}$ (full circles). Note the presence of a ferromagnetic transition. Results for empty $\mathrm{Cd}_{0.976} \mathrm{Mn}_{0.024} \mathrm{Te} \mathrm{QWs}$, where antiferromagnetic interactions dominate, are shown by empty symbols (after Haury et al. [14]).

susceptibility in the limit of vanishing fields was determined from

$$
\chi(T)=\left(g \mu_{\mathrm{B}} /|\alpha-\beta|\right)(\partial \Delta / \partial H) .
$$

The transition temperature deduced from the extrapolation of the susceptibility data shown in figure $2 \mathrm{~b}$ agreed well with the temperature deduced from the appearance of the spontaneous magnetization. The critical behaviour of $\chi(T)$ is unlike the gradual changes of $\chi(T)$ associated with the formation of magnetic polarons [40,47], for which the finite volume involved precludes the existence of any second order phase transition $[40,48]$.

These findings are interpreted as a ferromagnetic phase transition driven by the free holes which by means of the RKKY mechanism mediate ferromagnetic exchange interactions between the Mn spins. This conclusion is strongly supported by the theoretical model discussed above. According to that model [13], the critical fluctuations of magnetization are of minor importance, so that we may write $\chi(T)=$ $C /\left(T+T_{o}-\Theta\right)$, where the influence of the delocalized carriers is described by $\Theta$. According to the results of figure $2 \mathrm{~b}$, and in agreement with the model, the same 
values of the Curie constant $C$ are observed in the presence and in the absence of the holes. At the same time, again in accord with the model, $T_{\mathrm{c}}$ does not depend on $p$ (as long as holes are delocalized) but increases with $\tilde{x}$. From $T_{\mathrm{c}}$ and $\Theta=T_{\mathrm{c}}-T_{o}$ the enhancement factor $A_{\mathrm{F}}$ is obtained to be $2.5 \pm 0.5$, an expected value in view of the discussion presented in section 5 .

In addition to enhancing $\Theta$, static disorder and carrier-carrier interactions impose a strong influence on the optical properties. In particular, they lead to a band-gap narrowing [49] that amounts to $29 \mathrm{meV}$ for all doped samples studied, as deduced from the energy difference between the free exciton reflectivity for the empty QW and the PL in the presence of the holes $(7 \mathrm{meV}$ at $H=0)$, and from an exciton binding energy of $22 \mathrm{meV}$, according to an estimate based on the $1 s-2 s$ excitonic energy separation [50] in the undoped sample. Moreover, these renormalization effects are expected to vary strongly with the degree of carrier polarization. Accordingly, while the evaluated value of the splitting of the heavy hole subband corresponding to the full hole polarization, $\Delta \approx 6 \mathrm{meV}$ for $p=2 \times 10^{11} \mathrm{~cm}^{-2}$, is not inconsistent with the experimental results of figure 2 , the achievement of the saturation with the lowering of temperature proceeds slower than predicted by the model. A work is under way aimed at determining the effect of the renormalization on the dependence $\Delta(T, H)$ as well as at examining the domain structure in this novel ferromagnetic system.

\section{Conclusions and outlook}

The findings presented above demonstrate that p-type doping actually constitutes the method for substantial enhancement of magnetic effects, and then magnetooptical effects in DMS, leading - in appropriately designed structures - to a ferromagnetic phase transformation. Additional enhancement of the tendency towards the ferromagnetic ordering is possible by engineering such a microscopic distribution of the magnetic ions, which would reduce $T_{o}$ and/or increase $\Theta$. Since the interactions between localized spins mediated by the carriers are long range ones, a simple mean-field approach gives a correct quantitative description of magnetic properties, even in reduced dimensionality systems. At the same time, the data make it possible to evaluate the strength of many body effects for the case of two-dimensional hole gas. Moreover, while our results demonstrate the possibility of changing the magnetic phase by light, other means - such as gates - are expected to also provide a high degree of control over magnetic properties in modulation-doped structures. This opens new prospects for further studies of coupled carrier liquids and localized spins in novel geometries and material systems.

\section{Acknowledgements}

I would like to thank my principal co-workers from Grenoble Yves Merle d'Aubigné, Joel Cibert, and André Wasiela for fruitful and enjoyable collaboration. The work in Poland was supported by KBN Grant No. 2-P03B-6411, while the collaboration by Polish-French cooperation project "Polonium" and Joseph Fourier University. 


\section{References}

1. Dietl T. - In: Handbook on Semiconductors, edited by Moss T.S. (North-Holland, Amsterdam), 1994, vol. 3b, p. 1251; Furdyna J.K., J. Appl. Phys., 1988, vol. 64, p. R29.

2. Łazarczyk P., Story T., Arciszewska M., Gałązka R.R. // J. Magn. Magn. Materials, 1997, vol. 169, p. 151.

3. Koshihara S., Oiwa A., Hirasawa M., Katsumoto S., Iye Y., Urano C., Takagi H., Munekata H. // Phys. Rev. Lett., 1997, vol. 78, p. 4617; Ohno H., Shen A., Matsukura F., Oiwa A., Endo A., Katsumoto S., Iye Y. // Appl. Phys. Lett., 1996, vol. 69, p. 363.

4. Ohno H., Matsukura F., Shen A., Sugawara Y., Oiwa A., Endo A., Katsumoto S., Iye Y. - In: Proc. 23rd International Conference on the Physics of Semiconductors, Berlin 1996, edited by M. Scheffler and R. Zimmermann (World Scientific, Singapore, 1996) p. 405.

5. Matsukura F., Ohno H., Shen A., Sugawara Y. // Phys. Rev. B, 1998, vol. 57, p. R2037.

6. Blinowski J., Kacman P., Majewski J.A. // Phys. Rev. B, 1996, vol. 53, p. 9524.

7. Wojtowicz T., Karczewski G., Kossut J. // Thin Solid Films, 1997, vol. 306, p. 271.

8. Shibata N., Ohki A., Katsui A. // J. Cryst. Growth, 1988, vol. 93, p. 703.

9. Baron T., Tatarenko S., Saminadayar K., Magnea N., Fontenille J. // Appl. Phys. Lett., 1994, vol. 65, p. 1284.

10. Bassani F., Tatarenko S., Saminadayar K., Magnea N., Cox R.T., Tardot A., Grattepain C. // J. Appl. Phys., 1992, vol. 72, p. 2927; Scholl S., Schäfer H., Waag A., Hommel D., von Schierstedt K., Kuhn-Heinrich B., Landwehr G. // Appl. Phys. Lett., 1993, vol. 62, p. 3010; Smorchkova I., Samarth N. // Appl. Phys. Lett., 1996, vol. 69, p. 1640.

11. Tatarenko S., Baron T., Arnoult A., Cibert J., Grün M., Haury A., Merle d'Aubigné Y., Wasiela A., Saminadayar K. // J. Crystal Growth, 1997, vol. 175/176, p. 682; Arnoult A., Tatarenko S., Ferrand D., Cibert J., Haury A., Wasiela A., Merle d'Aubigné Y. // J. Crystal Growth, 1998, vol. 184/185, p. 445.

12. Jaroszyński J., Wróbel J., Sawicki M., Kamińska E., Skośkiewicz T., Karczewski G., Wojtowicz T., Piotrowska A., Kossut J., Dietl T. // Phys. Rev. Lett., 1995, vol. 75, p. 3170; Jaroszyński J., Wróbel J., Karczewski G., Wojtowicz T., Dietl T. // Phys. Rev. Lett., 1998, vol. 80, p. 5635.

13. Dietl T., Haury A., Merle d'Aubigné Y. // Phys. Rev. B, 1997, vol. 55, p. R3347.

14. Haury A., Wasiela A., Arnoult A., Cibert J., Tatarenko S., Dietl T., Merle d'Aubigné Y. // Phys. Rev. Lett., 1997, vol. 79, p. 511.

15. Cibert J., Kossacki P., Haury A., Wasiela A., Merle d'Aubigné Y., Dietl T., Arnoult A., Tatarenko S. // J. Crystal Growth, 1998, vol. 184/185, p. 898.

16. Merle d'Aubigné Y., Arnoult A., Cibert J., Dietl T., Haury A., Kossacki P., Tatarenko S., Wasiela A. // Physica E (in press).

17. Gaj J.A., Grieshaber W., Bodin-Deshayes C., Cibert J., Feuillet G., Merle d'Aubigné Y., Wasiela A. // Phys. Rev. B, 1994, vol. 50, p. 5512.

18. Kittel C. Solid State Physics, vol. 22, edited by Seitz F., Turnbull D., Ehrenreich H. New York, Academic Press, 1968, p. 1.

19. Ruderman M.A., Kittel C. // Phys. Rev., 1954, vol. 96, p. 99.

20. Yafet Y. // Phys. Rev. B, 1987, vol. 36, p. 3948. 
21. Aristov D.N. // Phys. Rev. B, 1997, vol. 55, p. 8064; see also, Korenblit I.Ya., Shender E.F. // Zh. Eksp. Teor. Fiz., 1975, vol. 69, p. 1113 [Sov. Phys. JETP, 1975, vol. 42, p. 566].

22. Leroux-Hugon P. - In: New Developments in Semiconductors, edited by Wallace P.R., Harris R., Zuckermann M.J. Leyden, Noordhoff, 1973, p. 63; Krivoglaz M.A. // Usp. Fiz. Nauk, 1973, vol. 111, p. 617 [Sov. Phys. Usp., 1974, vol. 16, p. 856].

23. Pashitskii E.A., Ryabchenko S.M. // Fiz. ]ver. Tela, 1979, vol. 21, p. 545 [Sov. Phys. Solid State, 1979, vol. 21, p. 322].

24. Fisher M.E., Ma S.-k., Nickel B.G. // Phys. Rev. Lett., 1972, vol. 29, p. 917.

25. Yeomans J.M. Statistical Mechanics of Phase Transitions. Oxford, Oxford University Press, 1993, p. 57.

26. Łazarczyk P., Dobrowolski W., Story T. - In: Extended Abstracts of International Conference on Electron Localization and Quantum Transport in Solids, Jaszowiec, Poland, 1996, edited by Dietl T. Warsaw, Institute of Physics, 1996, p. 129.

27. von Molnar S., Kasuya T. // Phys. Rev. Lett., 1968, vol. 21, p. 1757; von Molnar S., Shafer M.W. // J. Appl. Phys., 1970, vol. 41, p. 1093.

28. Wachter P. // CRC Crit. Rev. Solid State Sci., 1972, vol. 3, p. 189.

29. de Gennes P.G. // J. Phys. Radium, 1962, vol. 23, p. 630.

30. Zyuzin A.Yu., Spivak B.Z. // Pisma Zh. Eksp. Teor. Fiz., 1986, vol. 43, p. 185 [JEPT Lett., 1986, vol. 43, p. 234]; Bulaevskii L.N., Panyukov S.V. // Pisma Zh. Eksp. Teor. Fiz., 1986, vol. 43, p. 190. [JEPT Lett., 1986, vol. 43, p. 240]; Jagannathan A., Abrahams E., Stephen M.J. // Phys. Rev. B, 1988, vol. 37, p. 436.

31. Altshuler B.L., Aronov A.G. - In: Electron-Electron Interaction in Disordered Systems, edited by Efros A.L., Pollak M. Amsterdam, North-Holland, 1985, p. 1; Fukuyama H., ibid., p. 155; Lee P.A., Ramakrishman T.V. // Rev. Modern Phys., 1985, vol. 57, p. 287.

32. Altshuler B.L., Aronov A.G., Zyuzin A.Yu. // Pisma Zh. Eksp. Teor. Fiz., 1983, vol. 38, p. 128 [JETP Lett., 1983, vol. 38, p. 153].

33. Dietl T. - In: Ref. 1, pp. 1280, 1284.

34. Sawicki M., Dietl T., Kossut J., Igalson J., Wojtowicz T., Plesiewicz W. // Phys. Rev. Lett., 1986, vol. 56, p. 508; Shapira Y., Oliveira N.F., Becla Jr., P., Vu T.Q. // Phys. Rev. B, 1990, vol. 41, p. 5931; Jaroszyński J., Wróbel J., Sawicki M., Kamińska E., Skośkiewicz T., Karczewski G., Wojtowicz T., Piotrowska A., Kossut J., Dietl T. // Phys. Rev. Lett., 1995, vol. 75, p. 3170; Smorchkowa I.P., Samarth N., Kikkawa J.M., Awschalom D.D. // Phys. Rev. Lett., 1997, vol. 78, p. 3571.

35. Brunthaler G., Dietl T., Jaroszyński J., Sawicki M., Stöger G., Prinz A., Schäffler F., Bauer G. // Semicond. Sci. Techn., 1996, vol. 11, p. 1624.

36. Dietl T., Sawicki M., Isaacs E.D., Dahl M., Heiman D., Graf M.J., Gubariev S.I., Alov D.L. // Phys. Rev. B, 1991, vol. 43, p. 3154.

37. see, e.g., Bhatt R.N., Paalanen M.A., Sachdev S. // J. de Physique, 1988, vol. 49, p. C8-1179.

38. Głód P., Dietl T., Sawicki M., Miotkowski I. // Physica B, 1994, vol. 194-196, p. 995.

39. Egger R., Schoeller H. // Phys. Rev. B, 1996, vol. 54, No. 16, p. 337.

40. Dietl T., Spałek J. // Phys. Rev. Lett., 1982, vol. 48, p. 355; Phys. Rev. B, 1983, vol. 28 , p. 1548.

41. Le Si Dang, Neu G., Romestain R. // Solid State Commun., 1982, vol. 44, p. 1187.

42. Gaj J.A., Ginter J., Gałązka R.R. // Phys. Status Solidi (b), 1978, vol. 89, p. 655. 
43. We note that the formation of bound magnetic polarons shifts the delocalization transition towards higher carrier concentrations; see, e.g., Głód, Dietl T., Sawicki M., Miotkowski I. // Physica B, 1994, vol. 194-196, p. 995.

44. Novak M.A., Symko O.G., Zheng D.J., Oseroff S. // J. Appl. Phys., 1985, vol. 57, p. 3418.

45. Fishman G. // Phys. Rev. B, 1995, vol. 52, No. 11, p. 132.

46. Peyla P., Wasiela A., Merle d'Aubigné Y., Ashenford D.E., Lunn B. // Phys. Rev. B, 1993, vol. 47, p. 3783; Kuhn-Heinrich B., Ossau W., Bangert E., Waag A., Lanwehr G. // Solid State Commun., 1994, vol. 91, p. 413.

47. Wojtowicz T., Koleśnik S., Miotkowski I., Furdyna J.K. // Phys. Rev. Lett., 1993, vol. 70, p. 2317.

48. Benoit à la Guillaume C. // Phys. Stat. Solidi (b), 1993, vol. 175, p. 369.

49. Schmitt-Rink S., Chemla D.S., Miller D.A.B. // Adv. Phys., 1989, vol. 38, p. 89.

50. Mathieu H., Lefebre P., Christol P. // Phys. Rev. B, 1992, vol. 46, p. 4092. 


\section{Феромагнітний перехід у розведених магнітних напівпровідниках}

\section{Т.Діетль}

Інститут фізики і Коледж природничих наук Польської академії наук, Польща, PL-02668 Варшава, ал. Льотнікув, 32/46,

Отримано 6 липня 1998 р.

Зроблено огляд недавніх теоретичних робіт про вплив взаємодії Рудермана-Кіттеля-Касуї-Іоміди між локалізованими спінами у системах різної вимірності в розведених магнітних напівпровідниках (PMH). Оскільки ця взаємодія далекосяжна, її вплив на температурну і польову залежності намагніченості і спінове розщеплення зон оцінюється в наближенні середнього поля, беручи до уваги змінену безладом взаємодію носій-носій. Теоретичні оцінки показують, що експериментально досяжні густини дірок достатньо високі для того, щоб спричинити фазовий перехід парамагнетик-феромагнетик у тривимірних модульованих структурах II-VI PMH. Показано, що результати теоретичних обчислень підтверджуються недавніми магнетооптичними дослідженнями вирощених методом НВЕ зразків $\mathrm{Cd}_{1-x} \mathrm{H}_{n x} \mathrm{Te} / \mathrm{Cd}_{1-4-7} \mathrm{Hg}_{y} \mathrm{Zn}_{z}: \mathrm{N}$, що містять одну модульовану квантову яму глибиною $8 \mathrm{~nm}$. Ці дослідження свідчать про наявність феромагнітного переходу, спричиненого двовимірним дірковим газом. Перехід відбувається при температурі між 1.8 i $2.5 \mathrm{~K}$, залежно від концентрації марганцю $x$, що узгоджується з теоретичною моделлю.

Ключові слова: напівмагнітні напівпровідники, взаємодія RKKY, квантові ями

PACS: $75.50 . R r, 75.30 . H x, 75.50 . D d, 78.55 . E t$ 